\title{
Long-Term Outcomes of Recurrent Thymoma
}

Cai Xu1, Qinfu Feng ${ }^{1 *}$, Zhouguang Hui ${ }^{1}$, Chengcheng Fan ${ }^{2}$, Yirui Zhai' ${ }^{1}$, Yidong Chen ${ }^{3}$, Hongxing Zhang ${ }^{1}$, Zefen Xiao ${ }^{1}$, Jun Liang ${ }^{1}$, Dongfu Chen ${ }^{1}$, Zongmei Zhou ${ }^{1}$, Miaoli Zheng ${ }^{1}$, Xiaodan Wang ${ }^{1}$, Lvhua Wang ${ }^{1 *}$ and Jie $\mathrm{He}^{4}$

${ }^{1}$ Department of Radiation Oncology, Cancer Hospital/Institute, Chinese Academy of Medical Sciences and Peking Union Medical College, Beijing, 100021, China ${ }^{2}$ Department of Radiation Oncology, Cancer Hospital Affiliated to Zhengzhou University, Henan Province, China

${ }^{3}$ Department of Radiation Oncology, Beijing Shijitan Hospital, Beijing, China

${ }^{4}$ Department of Thorax Surgery, Cancer Hospital/Institute, Chinese Academy of Medical Sciences and Peking Union Medical College, Beijing, 100021, China

\begin{abstract}
Background: This study sought to analyze the results and prognosis of recurrent thymoma.

Methods: Between 1991 and 2012, 32 patients that developed recurring thymoma after radical resection at initial treatment were reviewed.

Results: The median follow-up duration after initial treatment and recurrence was 89 and 49.5 months, respectively. The median recurrence free internal (RFI) was 42 months, and the 5-year overall survival (OS) rates following recurrence was $65.5 \%$ for recurrent thymoma. Among 32 patients that relapsed, 7 underwent reoperation, 18 experienced nonsurgical management, 5 failed to receive treatment, and 2 remain unknown. The 5 -year OS rates after recurrence of the surgery plus adjuvant chemotherapy/radiotherapy group and the non-surgery group were $100 \%$ and $73.1 \%$, respectively $(P=0.210)$. Histological $\mathrm{WHO}$ upgrading was frequently observed $(57.1 \%)$ in patients with recurrent thymoma who received reoperation. In univariate analysis, age $(<55$ years, $P=0.009)$, local and regional recurrence $(P=0.022)$, and late recurrence $(R F I \geq 20$ months, $P=0.038)$ indicated good prognostic factors of recurrent thymoma.
\end{abstract}

Conclusions: Reoperation plus adjuvant chemotherapy/radiotherapy may result in good outcomes for patients who can tolerate surgery when complete resection is possible, and may get better results than non-surgery treatment. Histological WHO upgrade was frequently observed in recurrent thymoma. Age $<55$ years, local and regional recurrence, and longer RFI (RFI $\geq 20$ months) were associated with a positive prognosis.

Keywords: Recurrent thymoma; Retreatment; Outcome; Prognostic factor

\section{Background}

Surgery remains the main treatment of thymoma, and complete resection is the most important prognostic factor [1,2]. However, the relapse of thymoma is common even after radical resection, with a postcomplete resection recurrence rate ranging from $5 \%$ to $50 \%$ [3]. Owing to the low morbidity of thymoma, the lower incidence of recurrence, various recurrence patterns, retrospective single institute nature of studies, and the selective bias of studies, the optimal strategy for managing recurrent thymoma remains controversial. Most of authors have recommended reoperation for recurrent thymoma based on improved survival compared to non-operated patients [4-10], whereas a few have discouraged reoperation because of the high perioperative morbidity $[4,11,12]$. We reviewed retrospectively the outcomes and prognostic factors of 32 patients that relapsed after radical resection to try to bolster strategies for the treatment and surveillance of recurrent thymoma.

\section{Methods}

Institutional Review Board of Cancer Hospital and Institute, Chinese Academy of Medical Sciences approved this retrospective study; this being an observational study, no additional interventions were required.

\section{Patients and methods}

We retrospectively reviewed the medical records of 32 patients with recurrent thymoma who received complete resection at primary treatment in our hospital between 1991 and 2012. Patients with R1/ $\mathrm{R} 2$ resection or biopsy resection and thymic carcinoma were excluded. All patients agreed to participate in this research. The Masaoka-Koga staging systems [13] were adopted and pathologic results were classified according to WHO histological classification [14]. Recurrent thymoma and recurrence patterns were identified based on the International Thymic Malignancy Interest Group (ITMIG) definitions [15]. One oncologist and two radiologists diagnosed patients with recurrence according to clinical symptoms and the results of CT scan and MRI when needed. Biopsy was not mandatory.

\section{Initial treatment of thymoma}

Patient characteristics were summarized in Table 1 . All 32 patients with recurrence underwent radical surgery initially, among whom 17 received postoperative radiotherapy (PORT) with a median target dose of 5800 cGy (range, 4000 cGy to 6000 cGy) in 200 cGy fractions over 5-6 weeks; the dose of 2 patients was unknown. Of the 32 patients, only 1 patient received cisplatin/doxorubicin based chemotherapy for 8 cycles.

\section{Statistical analysis}

The rates of overall survival (OS) after initial treatment and OS

*Corresponding authors: Qin-Fu Feng, Department of Radiation Oncology Cancer Hospital/Institute, Chinese Academy of Medical Sciences, and Peking Union Medical College, Beijing, China, Tel: 0086-010-87788286; Fax; 0086-01062108145; E-mail: qinfufeng19@163.com

Lvhua Wang, Department of Radiation Oncology, Cancer Hospital/Institute, Chinese Academy of Medical Sciences, and Peking Union Medical College, Beijing-100021, China, Tel: 0086-010-8778799; Fax; 0086-010-62108145; E-mail: wlhwq@yahoo.com

Received July 09, 2016; Accepted September 15, 2016; Published September 19, 2016

Citation: Xu C, Feng Q, Hui Z, Fan C, Zhai Y, et al. (2016) Long-Term Outcomes of Recurrent Thymoma. J Pulm Respir Med 6: 367. doi: 10.4172/2161-105X.1000367

Copyright: ( 2016 Xu C, et al. This is an open-access article distributed under the terms of the Creative Commons Attribution License, which permits unrestricted use, distribution, and reproduction in any medium, provided the original author and source are credited. 
following recurrence were calculated with the Kaplan-Meier method. OS rates after initial treatment was measured from the date of operation until the day of death or last follow-up visit. OS rates following recurrence were measured from the date of recurrence until the day of death or last follow-up visit. Recurrence-free interval (RFI) was measured from the date of operation until the date of recurrence, death, or last follow-up visit. Univariate analysis was performed with the Kaplan-Meier method to assess prognostic factors for recurrent thymoma. Differences in categorical variables were statistically examined using the Fisher's exact test. A P-value less than 0.05 indicated statistical significance. Statistical analysis was performed using SPSS software version 19.0.

\section{Results}

\section{Recurrence sites and patterns}

Among 32 patients, relapses were found in the following sites: pleura (21 cases, 66\%), tumor bed (10.31\%), lung (7.22\%), chest wall (6.19\%), lymph node metastasis (4.13\%), non-tumor bed in mediastinum (1), abdominal node metastasis (1), liver (1), and overlapped recurrence (13.41\%). According to the ITMIG classification, the patterns of recurrence were shown in Figure 1.

\section{Survival}

The median follow-up duration after initial treatment and recurrence was 89 months (range, 15-253 months) and 49.5 months (range, 1-136 months), respectively. The 5-year and 10-year OS rates for the entire group after initial treatment were $83.6 \%$ and $66.5 \%$,

\begin{tabular}{|c|c|}
\hline Characteristics & No. (\%) \\
\hline \multicolumn{2}{|l|}{ Sex } \\
\hline Male/female & $19(59) / 13(41)$ \\
\hline Median age at diagnosis, year (range) & $45(30-68)$ \\
\hline Median tumor size, cm (range) & $8(4-22) / 2$ Unknown \\
\hline \multicolumn{2}{|l|}{ Myasthenia gravis at detection } \\
\hline Present/absent & $6(19) / 26(81)$ \\
\hline \multicolumn{2}{|l|}{ Myasthenia gravis at recurrence } \\
\hline Present/absent & $4(13) / 28(87)$ \\
\hline \multicolumn{2}{|l|}{ Masaoka stage at initial detection } \\
\hline 1 & $3(9)$ \\
\hline II & $14(44)$ \\
\hline III & $10(31)$ \\
\hline IV & $5(16)$ \\
\hline \multicolumn{2}{|l|}{ WHO classification at first detection } \\
\hline$A+A B$ & $5(16)$ \\
\hline B1 & $7(22)$ \\
\hline B2 & $6(19)$ \\
\hline B3 & $12(37)$ \\
\hline Unknown & $2(6)$ \\
\hline \multicolumn{2}{|l|}{ Adjuvant treatment after first surgery } \\
\hline Postoperative chemotherapy & $1(3)$ \\
\hline Postoperative radiotherapy & $17(53)$ \\
\hline No postoperative treatment & $14(44)$ \\
\hline \multicolumn{2}{|l|}{ Treatment } \\
\hline Surgery & $7(22 \%)$ \\
\hline Nonsurgery & $18(56 \%)$ \\
\hline Radiotherapy & $9(28)$ \\
\hline Chemotherapy & $6(19)$ \\
\hline Chemoradiotherapy & $3(9)$ \\
\hline None & $5(16)$ \\
\hline Missing & $2(6)$ \\
\hline Median recurrence-free interval, month (range) & $42(5-193)$ \\
\hline
\end{tabular}

Table 1: Characteristics of the 32 patients with recurrent thymoma.

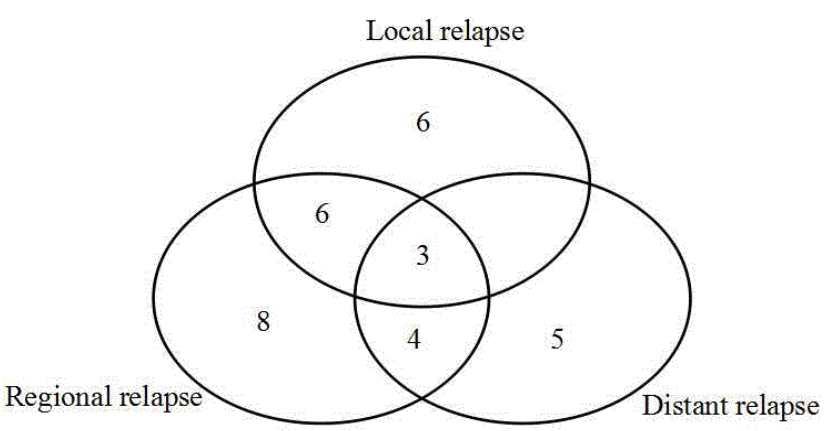

Figure 1: Patterns of relapse.

respectively, and the 5-year OS rate after recurrence was $65.5 \%$. Only one patients died from nontumor related cause. Tumor specific OS rate following recurrence was $67.7 \%$. Among 32 patients with recurrence, 14 showed a single recurrence and 18 demonstrated multiple recurrence, and the 5- and 10-year OS rates after recurrence were $75.2 \%$ and $57.1 \%$, respectively ( $\mathrm{P}=0.668)$.

\section{The outcomes of recurrent thymoma}

Table 2 shows the outcomes of 32 patients that relapsed. Seven patients with recurrence received reoperation, including $5 \mathrm{R} 0$ resection and 2 R2 resection, and the rate of complete resection was $71 \%$. Three patients with regional recurrence received R0 resection, two of whom had single recurrence and received postoperative radiotherapy or chemotherapy. One patients with local and regional recurrence received $\mathrm{R} 2$ resection and postoperative chemoradiotherapy because of the extensive diseases and multiple sites. One patients with regional and distant (lung) recurrence received R0 resection and postoperative chemoradiotherapy. Two patients had local, regional and distant recurrence, one of whom received total pleural pnemonectomy and got R0 resection, the other received partial mediastinal tumor resection and postoperative radiotherapy. Three patients (43\%) displayed a second recurrence and received second treatment. The 5-year OS between the surgery group plus adjuvant chemotherapy/radiotherapy and the nonsurgery group was not statistic significant $(\mathrm{P}=0.210)$.

Among 9 patients that received radiotherapy, 4 had local recurrence and 5 had regional or distant recurrence. The median survival interval following recurrence of the local recurrence group and the regional or distant recurrence group was 60 months (range, 51-107 months) and 37 months (range, 23-106 months), respectively. The 5-year OS rates following recurrence of the local recurrence group and the regional or distant recurrence group were $100 \%$ and $60 \%$, respectively $(\mathrm{P}=0.34)$.

Six patients with multiple sites received cisplatin/doxorubicinbased chemotherapy (cisplatin $75 \mathrm{mg} / \mathrm{m}^{2}$, iv day2; doxorubicin $50 \mathrm{mg}$ / $\mathrm{m}^{2}$, iv, day1, repeated 21 days) for 6-8 cycles, three of whom had local and regional recurrence, one had regional recurrence, one had regional and distant (lung) recurrence, and one had local, regional and distant recurrence. Three patients received sequential chemoradiotherapy with local, local and regional, and regional recurrence, respectively.

Five patients did not receive any treatment after recurrence, 3 of whom had distant recurrence (1 lung), one had local recurrence, and one had regional and distant (lung) recurrence. The 2 -year OS rates after recurrence of the groups of patients receiving retreatment, nonsurgical management, and radiotherapy were better than the group of patients without retreatment, respectively $(\mathrm{P}<0.05)$. The 2 -year OS rate and the 


\begin{tabular}{|l|c|c|c|c|c|}
\hline Events & NO. (\%) & Median age (y) & Median RFI (m) & Median survival (m) \\
\hline Surgery & $7(22)$ & 45 & 43 & 5y-OS (\%) \\
\hline Nonsurgery & $18(56)$ & 41.5 & 40.5 & $55(6-107)$ \\
\hline RT & $9(28)$ & 37 & 41 & 700 \\
\hline CT & $6(19)$ & 45.5 & 9.5 & 79.1 \\
\hline CRT & $3(9)$ & 50 & 121 & $30(6-86)$ \\
\hline WT & $5(16)$ & 63 & 30 & $66(57-85)$ \\
\hline Unknown & $2(6)$ & 35 & 73 & 37.5 \\
\hline
\end{tabular}

OS: Overall Survival; RFI: Recurrence Free Interval; RT: Radiotherapy; CT: Chemotherapy; CRT: Chemoradiotherapy; WT: Without Treatment; Y: Years; M: Months.

Table 2: The outcomes of recurrent thymoma.

median survival interval after recurrence of the chemotherapy group was higher compared with the group of patients without retreatment, but no statistical significance $(\mathrm{P}=0.062)$.

\section{The advance of WHO histological types}

Seven patients exhibited pathologic results after recurrence, among which $4(57.1 \%)$ had advanced WHO types, with 1 type $\mathrm{AB}$ to B2, 1 type B2 to B3, 1 type B1 to B2, and 1 type B3 to type C. There were no difference in age, sex, tumor size, Masaoka stage at initial detection, WHO types at initial detection, initial treatment, RFI, and the prognosis of 4 patients with advanced WHO types and 3 patients without advanced WHO types, respectively $(\mathrm{P}>0.05)$.

\section{Retreatment-related complication}

Adverse events were analyzed according to the National Cancer Institute Common Terminology Criteria for Adverse Events version 4.0. There was no treatment-related death or myasthenia gravis-related death after recurrence. Among 7 patients with re-resection, only 1 had postoperative infection. Among 9 patients that received radiotherapy, only 1 showed radiation pneumonitis of grade 2 . Among 9 patients that obtained chemotherapy or chemoradiotherapy, 5 patients (55.6\%) demonstrated gastrointestinal adverse reaction of grade 3 or less, and 1 displayed myelosuppression of grade 4 who recovered after treatment.

\section{The prognostic factors of recurrent thymoma}

The OS curves following recurrence of PORT at primary treatment, age at initial detection, WHO types at initial detection, Masaoka stage at initial detection, tumor size at initial detection, patterns of recurrence, single recurrence, and RFI were shown in Figure 2. In univariate analysis, age $(<55$ years, $\mathrm{P}=0.009)$, local and regional recurrence $(\mathrm{P}=0.022)$, and late recurrence $(\mathrm{RFI} \geq 20$ months, $\mathrm{P}=0.038$ ) were good prognostic factors of recurrent thymoma. However, Masaoka stage at initial detection, WHO types at initial detection, tumor size at initial detection, PORT at primary treatment, and single recurrence was not associated with survival of recurrent thymoma $(\mathrm{P}>0.05)$.

\section{Discussion}

Our results suggest that reoperation plus adjuvant chemotherapy/ radiotherapy may result in good outcomes for patients with local, regional, or distant (lung) recurrence whoever can tolerate surgery when complete resection is possible, and may get better results than non-surgery treatment, although no statistical significance which might be because of the small sample number. There may be statistical significance accompanied by the increase of samples. Our data is consistent with the outcomes of previous studies that reoperation might result in better survival than non-surgery treatment, especially when complete resection was possible $[3,4,6-8,10,12,16-21]$. A meta- analysis including 11 studies reported that surgical resection may be associated with improved long-term survival and should be considered for patients with recurrent thymoma [20]. Whereas, whether all of patients with recurrent thymoma should receive reoperation? The answer probably is no. It was reported that the 5-year OS rates after initial treatment of complete resection were much better than incomplete resection for patients with recurrent thymoma (64\% to $91.7 \%$ vs. $16 \%$ to $81.6 \%$ ) $[6,7,16,17]$, and complete resection even resulted in comparative survival with patients without recurrence [16], however, incomplete resection had no survival benefit and the prognosis was poor $[6,12,16,17]$. In addition, lung recurrences may be operated if they appear to be completely operable, which may achieve long-term survival [22]. And current evidence in the literature on the survival after debulking surgery for thymoma was contradictory, and most of the studies did not show any survival benefit after debulking for thymoma [23]. Furthermore, complete resection may have good survival even after the second recurrence [16]. Thus, reoperation may be recommended for recurrent thymoma whenever complete resection is possible. However, the rate of complete resection was low in previous studies $(50 \%$ to $90 \%)[4,6-8,16,17,24]$, which is consistent with our data. And we found that single recurrence had higher complete resection rate than multiple recurrence. Long-term observation $(\geq 10$ years) is necessary even for patients with radical resection and early stage diseases in order to detect recurrent disease as early as possible so that complete resection can be given [25].

And we also found that total pleural pneumonectomy resection may be feasible and safe for patients with localized unilateral pleural and/or lung relapse sites when complete resection was feasible. There was a patient in our study who had local, regional and distant recurrence, and extensive disease and multiple sites localized in unilateral thorax, received total pleural pnemonectomy without severe postoperative complication and got R0 resection, and he didn't experience relapse after 94 months. Gonzalez et al. [26] also reported the same results by reviewing 4 patients with stage IVA thymic tumor who underwent extrapleural pneumonectomy and thymectomy. However, Fabre et al. [27] reviewed retrospectively 17 patients with stage IVA thymoma who underwent a pleuropneumonectomy, and they found that pleuopneumonectomy was associated with a high morbid-mortality rate, whereas, it may provide good long-term survival with a multimodality strategy in highly selected patients. Pleuopneumonectomy may be considered as a treatment of choice when complete resection is possible, however, it need to be conducted conservatively under experience institutes because of the high morbidity of the procedure.

Whereas, unlike the previous studies [3,4,6-8,12,16-21], we observed that much high percent $(71.4 \%)$ of patients who underwent reoperation received adjuvant chemotherapy, radiotherapy, or chemoradiotherapy, which may improve the treatment outcomes of recurrent thymoma and confound the benefit of reoperation. Based on that adjuvant treatment can improve the outcomes of patients who have 

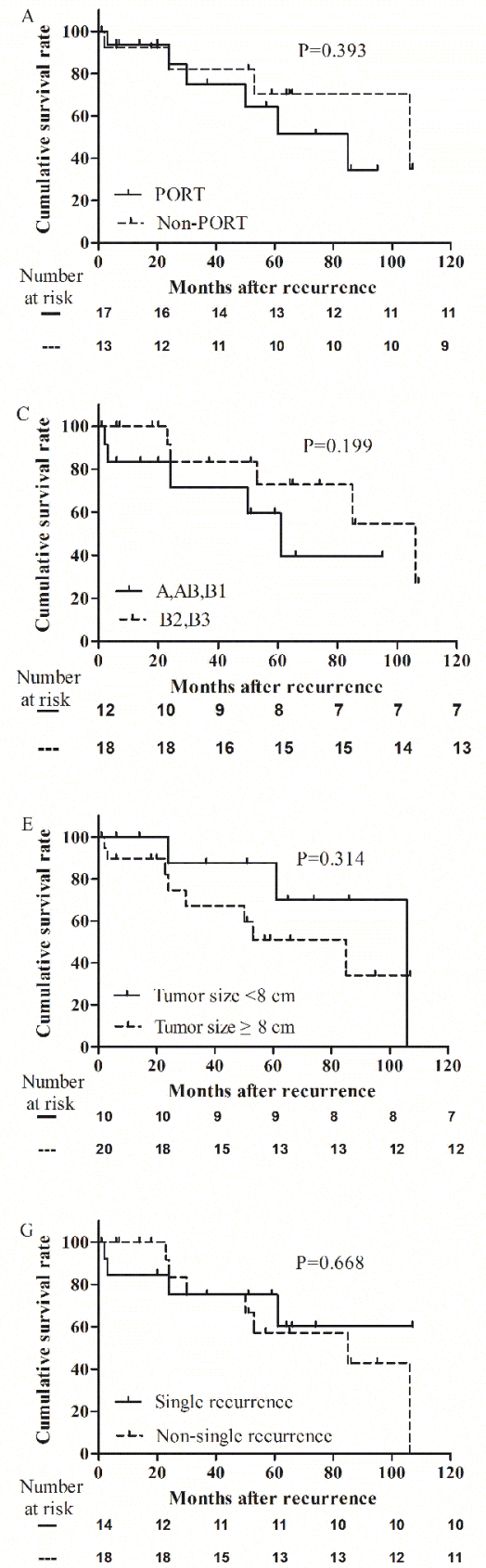
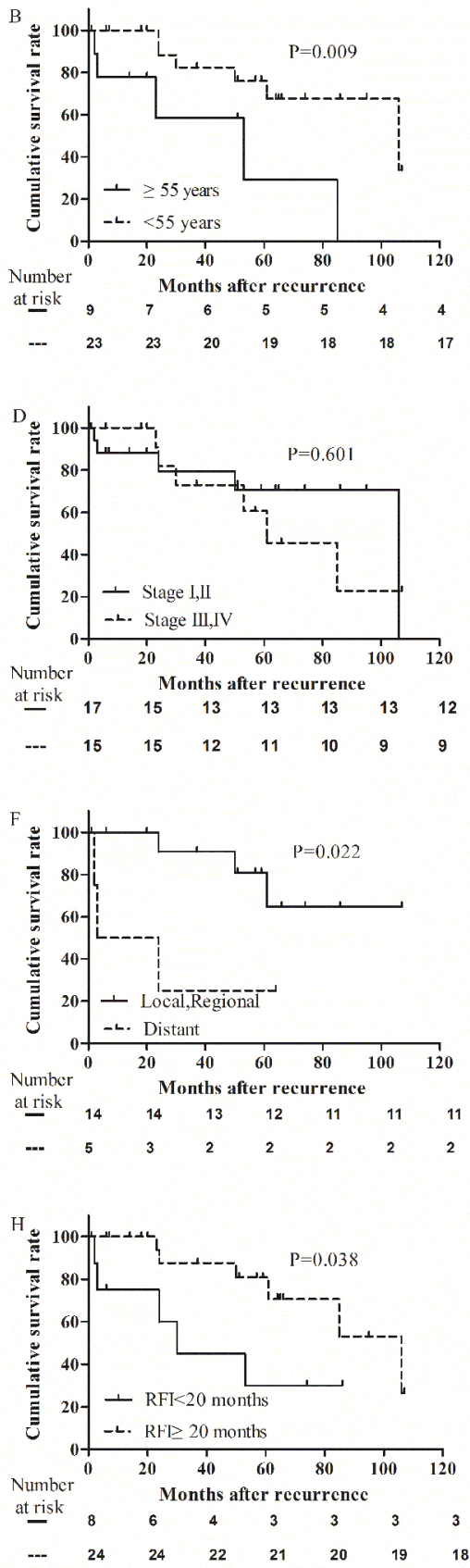

Figure 2: Overall survival difference after recurrence according to PORT. A: age; B: WHO histological type at initial; C: detection; D: Masaoka stage at initial detection; E: tumor size; F: patterns of recurrence; G: single recurrence; H: RFI.

advanced stage (III/IV) thymoma or incomplete resection [4,8,28-32], theoretically, adjuvant treatment may also improve the outcomes of recurrent thymoma. The good outcomes of the surgery group in our study may suggest that adjuvant treatment might improve the outcomes of patients with recurrence because of the high rate of patients receiving adjuvant treatment. However, a multicenter retrospective study reported that adjuvant chemotherapy or radiotherapy had no effect on prolonging the disease-free survival [24], however, the sample of this study was still small, and the percent of patients who received adjuvant treatment was low (around 20\%), which may confound the benefits of adjuvant treatment. Reoperation with curative intent associated with adjuvant therapy may be considered for patients with recurrent thymoma, including distant recurrences [33]. However, evidence on this is still lacking. More studies are needed to answer this question.

We found that non-surgery treatment (chemotherapy, radiotherapy, and chemoradiotherapy) may be considered when diseases were 
inoperable because of the severe medical complication or when complete resection was impossible because of the extent of the disease since the sensitivity of thymoma to chemotherapy and radiotherapy. In our study, the 5-year OS following recurrence of patients who received non-surgery treatment was $73.5 \%$, which was comparable with that of previous studies which included patients with surgery and non-surgery $(37 \%$ to $64 \%)[6-8,12,16,17]$. However, select bias might affect the reliability of the result.

Comparative outcomes of radiotherapy and chemotherapy of recurrent thymoma are lacking. We found that the survival of the radiotherapy group was higher than that of the chemotherapy group, although no statistical significance, which might be due to the selective bias of the study or the small sample number. 5-year survival rates of $65 \%$ with radiotherapy alone were obtained in small retrospective series of recurrent thymoma [34]. Definitive radiotherapy may be appropriate either alone or in combination with chemotherapy in inoperable localized recurrences [19]. And cisplatin-based chemotherapy may be appropriate either alone or in combination with radiotherapy or surgery in inoperable and relatively extensive diseases $[19,35,36]$. It is estimated that $50 \%-70 \%$ of patients with recurrent thymoma are suitable for chemotherapy [37]. The objective response rates and prolonged survival could be achieved with cisplatin based chemotherapy for metastatic or recurrent thymoma [38,39]. In the current study, patients received cisplatin/doxorubicin-based chemotherapy and had a relatively good survival. The chemotherapy drugs used in retreatment can be the same as the initial treatment, which are mainly cisplatin/doxorubicin-based chemotherapy regimens [38-41]. The combination of octreotide and prednisolone may be considered as one of the choices of treatment in patients with refractory recurrent thymoma [42]. The limited sample number and the significant bias of selection in the treatment might affect the reliability of the results, and we expect more studies with larger sample number.

This study suggests that recurrent thymoma may be needed to be treated actively because of the much poorer survival of patients without retreatment and the relative better survival of patients who received retreatment. In addition, retreatment is relatively safe based on the outcome of retreatment-related complication in this study. However, patients with older age and more advanced diseases tend to not receive retreatment, and patients showing recurrence might have long survival owing to the indolent feature of thymoma, which may affect the liability of the results, and overtreatment should be avoided.

The current study showed a high histological WHO upgrading rate (57.1\%). This phenomenon was also reported by previous studies, and the histological WHO upgrading rate was about $41 \%$ of recurrent thymoma patients [24,43-45]. The reason why WHO types might advance and whether the updating histology might affect prognosis of recurrent thymoma remains unclear. We found that the updating histology was not associated with prognosis of recurrent thymoma. And Sandri et al. [24] also reported the same results. In addition, the large ITMIG database with 4221 patients reported that WHO histology did not affect survival [46]. However, a small retrospective study reported that the upgrading histology may be associated with more advanced stage and worse prognosis [45]. More studies need to be conducted to address this issue.

It was reported that, type $\mathrm{AB}$ or $\mathrm{B} 1$, complete re-resection, local recurrence, the number of recurrent sites, and late recurrence were associated with good survival of recurrent thymoma, among which complete re-resection was the most important $[6,7,10,11,16]$. However, Masaoka stage at initial detection, PORT at primary treatment, the number of recurrent sites, MG, local and regional recurrence, and RFI have no association with the survival of recurrent thymoma $[6,12,16]$. Our data is partially consistent with the previous data that late recurrence (RFI $\geq 20$ months) and local and regional recurrence were good prognositic factors for recurrent thymoma, whereas, Masaoka stage at initial detection, PORT at initial treatment, and single recurrence had no statistically significant impact on survival of recurrent thymoma $(\mathrm{P}>0.05)$. And we also found that younger age $(<55$ years) was associated with a positive prognosis.

The first strength of this study was that we only included patients with recurrent thymoma who received complete resection at initial treatment, whereas, some of the previous studies included patients with thymoma and thymic carcinoma or patients with complete and incomplete resection at initial treatment, which may confound the treatment outcomes of thymoma. The second strength was that this study exhibited the most detailed analysis of the outcomes of recurrent thymoma. However, our study does have some intrinsic limitations. First, the current study, like previous ones, remains retrospective and derives from a single institution. Second, since thymomas relapse a long time after resection, and are slow growing, most patients did not reach the end of follow-up, and missing data is a source of significant bias. Third, the number of recurrent thymomas was extremely small and the study covered multiple decades in order to include a reasonable number of patients. Our study subjects were from single institute and the sample was really small, so it is not known whether our results are applicable to other groups. Further studies are needed in the future.

\section{Conclusion}

Reoperation plus adjuvant chemotherapy/radiotherapy may result in good outcomes for patients who can tolerate surgery when complete resection is possible, and may get better results than non-surgery treatment. Pleuopneumonectomy may be considered conservatively as a treatment of choice when complete resection is possible in experience institutes because of the high morbidity of the procedure. Non-surgery treatment may be considered when diseases were inoperable because of the severe medical complication or when complete resection was impossible because of the extent of the disease. Definitive radiotherapy may be appropriate either alone or in combination with chemotherapy in inoperable localized recurrences. Chemotherapy may be appropriate either alone or in combination with radiotherapy in inoperable and relatively extensive diseases. Histological WHO upgrade was frequently observed in recurrent thymoma. Age $<55$ years, local and regional recurrence, and longer RFI (RFI $\geq 20$ months) were associated with a positive prognosis.

\section{References}

1. Margaritora S, Cesario A, Cusumano G, Meacci E, D’Angelillo R, et al. (2010) Thirty-five-year follow-up analysis of clinical and pathologic outcomes of thymoma surgery. Ann Thorac Surg 89: 245-252.

2. Davenport E, Malthaner RA (2008) The role of surgery in the management of thymoma: a systematic review. Ann Thorac Surg 86: 673-684.

3. Lucchi M, Mussi A (2010) Surgical treatment of recurrent thymomas. J Thorac Oncol 5: S348-S351.

4. Bott MJ, Wang H, Travis W, Riely GJ, Bains M, et al. (2011) Management and outcomes of relapse after treatment for thymoma and thymic carcinoma. Ann Thorac Surg 92: 1984-1991.

5. Blumberg D, Port JL, Weksler B, Delgado R, Rosai J, et al. (1995) Thymoma: a multivariate analysis of factors predicting survival. Ann Thorac Surg 60: 908-913.

6. Ruffini E, Mancuso M, Oliaro A, Casadio C, Cavallo A, et al. (1997) Recurrence of thymoma: analysis of clinicopathologic features, treatment, and outcome. J Thorac Cardiovasc Surg 113: 55-63. 
7. Regnard JF, Zinzindohoue F, Magdeleinat $P$, Guibert L, Spaggiari L, et al. (1997) Results of re-resection for recurrent thymomas. Ann Thorac Surg 64: 1593-1598.

8. Hamaji M, Allen MS, Cassivi SD, Nichols FC, Wigle DA, et al. (2012) The role of surgical management in recurrent thymic tumors. Ann Thorac Surg 94: 247-254.

9. Okumura M, Shiono H, Inoue M, Tanaka H, Yoon HE, et al. (2007) Outcome of surgical treatment for recurrent thymic epithelial tumors with reference to world health organization histologic classification system. J Surg Oncol 95: 40-44.

10. Marulli G, Margaritora S, Lucchi M, Cardillo G, Granone P, et al. (2016) Surgical treatment of recurrent thymoma: is it worthwhile? Eur J Cardiothorac Surg 49 : 327-332.

11. Yano M, Sasaki H, Moriyama S, Hikosaka Y, Yokota K, et al. (2011) Number of recurrent lesions is a prognostic factor in recurrent thymoma. Interact Cardiovasc Thorac Surg 13: 21-24.

12. Haniuda M, Kondo R, Numanami H, Makiuchi A, Machida E, et al. (2001) Recurrence of thymoma: clinicopathological features, re-operation, and outcome. J Surg Oncol 78: 183-188.

13. Koga K, Matsuno Y, Noguchi M, Mukai K, Asamura H, et al. (1994) A review of 79 thymomas: modification of staging system and reappraisal of conventional division into invasive and non-invasive thymoma. Pathol Int 44: 359-367.

14. Muller-Hermelink HK, Strobel P, Zettl A (2004) Combined thymic epithelia tumors. In: Travis WD, Brambilla E, Muller-Hermelink HK, Harris CC, editors. Pathology and genetics: tumours of the lung, pleura, thymus and heart (WHO classification of tumours). Lyon: IARC Press, France, pp: 196-201.

15. Huang J, Detterbeck FC, Wang Z, Loehrer PJ (2010) Standard outcome measures for thymic malignancies. J Thorac Oncol 5: 2017-2023.

16. Bae MK, Byun CS, Lee CY, Lee JG, Park IK, et al. (2012) Clinical outcomes and prognosis of recurrent thymoma management. J Thorac Oncol 7: 1304-1314.

17. Margaritora S, Cesario A, Cusumano G, Lococo F, Porziella V, et al. (2011) Single-centre 40 -year results of redo operation for recurrent thymomas. Eur Cardiothorac Surg 40: 894-900.

18. Ruffini E, van Raemdonck D, Detterbeck F, Rocco G, Thomas $P$, et al. (2011) Management of thymic tumors: a survey of current practice among members of the European Society of Thoracic Surgeons. J Thorac Oncol 6: 614-623.

19. Girard N, Mornex F, van Houtte P, Cordier JF, van Schil P (2009) Thymoma: a focus on current therapeutic management. J Thorac Oncol 4: 119-126.

20. Hamaji M, Ali SO, Burt BM (2014) A meta-analysis of surgical versus nonsurgical management of recurrent thymoma. Ann Thorac Surg 98: 748-755.

21. Marulli G, Lucchi M, Margaritora S, Cardillo G, Mussi A, et al. (2011) Surgical treatment of stage III thymic tumors: a multi-institutional review from four Italian centers. Eur J Cardiothorac Surg 39: e1-e7.

22. Hirono M, Nonaka M, Himuro N, Tomita Y, Kataoka D, et al. (2014) Two cases of thymoma with pulmonary metastasis: a case report. World J Surg Oncol 12: 114.

23. Attaran S, Acharya M, Anderson JR, Punjabi PP (2012) Does surgical debulking for advanced stages of thymoma improve survival? Interact Cardiovasc Thorac Surg 15: 494-497.

24. Sandri A, Cusumano G, Lococo F, Alifano M, Granone P, et al. (2014) Longterm results after treatment for recurrent thymoma: a multicenter analysis. J Thorac Oncol 9: 1796-1804.

25. Awad WI, Symmans PJ, Dussek JE (1998) Recurrence of stage I thymoma 32 years after total excision. Ann Thorac Surg 66: 2106-2108.

26. Gonzalez M, Krueger T, Perentes JY, Matzinger O, Peters S, et al. (2011) Extrapleural pneumonectomy with venous confluence resection for stage IVA thymic tumors. Ann Thorac Surg 91: 941-943.

27. Fabre D, Fadel E, Mussot S, Mercier O, Petkova B, et al. (2011) Long-term outcome of pleuropneumonectomy for Masaoka stage IVa thymoma. Eur J Cardiothorac Surg 39: e133-e138.

28. Spaggiari L, Casiraghi M, Guarize J (2012) Multidisciplinary treatment of malignant thymoma. Curr Opin Oncol 24: 117-122.
29. Kunitoh H, Tamura T, Shibata T, Takeda K, Katakami N, et al. (2010) A phase II trial of dose-dense chemotherapy, followed by surgical resection and/or thoracic radiotherapy, in locally advanced thymoma: report of a Japan Clinical Oncology Group trial (JCOG 9606). Br J Cancer 103: 6-11.

30. Huang J, Rizk NP, Travis WD, Seshan VE, Bains MS, et al. (2007) Feasibility of multimodality therapy including extended resections in stage IVA thymoma. J Thorac Cardiovasc Surg 134: 1477-1483.

31. Lombe DC, Jeremic B (2015) A Review of the Place and Role of Radiotherapy in Thymoma. Clin Lung Cancer 16: 406-412.

32. Gao L, Wang C, Fang W, Zhang J, Lv C, et al. (2013) Outcome of multimodality treatment for 188 cases of type B3 thymoma. J Thorac Oncol 8: 1329-1334.

33. Laperuta P, Napolitano F, Garzi A, Amato B, Vatrella A, et al. (2014) Extrathoracic recurrence of type A thymoma. Int J Surg 12: S16-S18.

34. Urgesi A, Monetti U, Rossi G, Ricardi U, Maggi G, et al. (1992) Aggressive treatment of intrathoracic recurrences of thymoma. Radiother Oncol 24: 221-225.

35. Falkson CB, Bezjak A, Darling G, Gregg R, Malthaner R, et al. (2009) The management of thymoma: a systematic review and practice guideline. J Thorac Oncol 4: 911-919.

36. Rajan A, Giaccone G (2011) Chemotherapy for thymic tumors: induction consolidation, palliation. Thorac Surg Clin 21: 107-111.

37. Huang J, Detterbeck FC, Wang Z, Loehrer PJ (2011) Standard outcome measures for thymic malignancies. J Thorac Oncol 6: S1691-S1697.

38. Loehrer PJ, Kim K, Aisner SC, Livingston R, Einhorn LH, et al. (1994) Cisplatin plus doxorubicin plus cyclophosphamide in metastatic or recurrent thymoma: final results of an intergroup trial. The Eastern Cooperative Oncology Group, Southwest Oncology Group, and Southeastern Cancer Study Group. J Clin Oncol 12: 1164-1168.

39. Giaccone G, Ardizzoni A, Kirkpatrick A, Clerico M, Sahmoud T, et al. (1996) Cisplatin and etoposide combination chemotherapy for locally advanced or metastatic thymoma. A phase II study of the European Organization for Research and Treatment of Cancer Lung Cancer Cooperative Group. J Clin Oncol 14: 814-820.

40. Girard N (2012) Chemotherapy and targeted agents for thymic malignancies. Expert Rev Anticancer Ther 12: 685-695.

41. Lara PN, Bonomi PD, Faber LP (1996) Retreatment of recurrent invasive thymoma with platinum, doxorubicin, and cyclophosphamide. Chest 110: 11151117.

42. Ito J, Sekiya M, Miura K, Yoshimi K, Suzuki T, et al. (2009) Refractory recurrent thymoma successfully treated with long-acting somatostatin analogue and prednisolone. Intern Med 48: 1061-1064.

43. Ike O, Wada H, Hitomi S (1991) Histopathological changes in invasive thymoma in patients with recurrence. Thorac Cardiovasc Surg 39: 228-231.

44. Ciccone AM, Rendina EA (2005) Treatment of recurrent thymic tumors. Semin Thorac Cardiovasc Surg 17: 27-31.

45. Pescarmona E, Rendina EA, Venuta F, Ricci C, Baroni CD (1995) Recurren thymoma: evidence for histological progression. Histopathology 27: 445-449.

46. Weis CA, Yao X, Deng Y, Detterbeck FC, Marino M, et al. (2015) The impact of thymoma histotype on prognosis in a worldwide database. J Thorac Oncol 10: $367-372$ 